\title{
Das Werk Heimito von Doderers, übersetzt in 30 Sprachen - eine Bilanz
}

\begin{abstract}
Werke von Heimito von Doderer wurden bisher in 30 Sprachen übersetzt. Der Beitrag gibt auf Grundlage der 2016 erschienenen „Bibliographie der in Übersetzung erschienenen Werke von Heimito von Doderer“ einen einführenden Überblick über die aktuelle Übersetzungslage. Die Auswertung der Bibliographie konzentriert sich auf die Vermittlung der Übersetzungshistorie von Doderers Euvre und auf die daraus resultierenden Schwerpunkte und Auffälligkeiten bei einzelnen Werken.
\end{abstract}

Keywords: Heimito von Doderer, Bibliographie, Übersetzung, Roman, Erzählung, Kurzgeschichte

Werke von Heimito von Doderer wurden bisher in 30 Sprachen übersetzt. Der nachfolgende Beitrag versucht auf Grundlage einer jüngst erschienenen Bibliographie einen einführenden Überblick über die diesbezügliche Übersetzungslage zu geben und andererseits, soweit das in einem solchen begrenzten Rahmen möglich ist, Ansätze für deren Auswertung zu vermitteln. Wie es der Zusammensetzung von Doderers Euvre entspricht, handelt es sich bei den übersetzten Texten des Autors in erster Linie um Romane, Erzählungen und Kurzgeschichten, gelegentlich auch aber um Gedichte und Essays. Die Tatsache einer Übertragung in 30 Sprachen zeigt, dass sein Werk eine doch sehr respektable internationale Verbreitung erfahren hat. Im Einzelnen waren das: Afrikaans, Arabisch, Bulgarisch, Chinesisch, Englisch, die Plansprache Esperanto, Estnisch, Finnisch, Französisch, Georgisch, Italienisch, Japanisch, Kirgisisch, Litauisch, Niederländisch, Norwegisch, Polnisch, Portugiesisch, Rumänisch, Russisch, Schwedisch, Serbokroatisch (B/K/S), Slowakisch, Slowenisch, Spanisch, Tschechisch, Türkisch, Ungarisch, Urdu und Weißrussisch. Läge das Gesamtwerk des Autors in jeder der genannten 30 Sprachen vor, so wäre Doderer unbestreitbar Teil der Weltliteratur. Ich bin zwar der Meinung, dass er auf jeden Fall dazu gezählt werden sollte, doch lässt sich über meine Beurteilung sicherlich streiten, nicht zuletzt weil von ihm jeweils nur wenige Seiten in so wichtigen Sprachen wie Arabisch, Chinesisch und Urdu vorliegen.

Zugleich kann nicht mit Sicherheit ausgeschlossen werden, dass Doderer noch in weitere Sprachen übersetzt wurde, denn es handelt sich bei den hier angeführten lediglich um jene Zielsprachen, für die ich im Rahmen meiner Arbeit an 
der „Bibliographie der in Übersetzung erschienenen Werke von Heimito von Doderer“ Nachweise und/oder Belege ermitteln konnte. In ihr werden daher zwar alle Übersetzungen genannt, die zu meiner Kenntnis gelangt sind, doch ist das allein keineswegs eine Garantie dafür, dass an entlegenen Orten nicht noch weitere publiziert wurden. Sie erhebt dementsprechend keinen Anspruch auf Vollständigkeit, weder im Hinblick auf die Zielsprachen noch auf die existierenden Übersetzungen.

Ursächlich hierfür sind vor allem zwei Umstände: Es existiert leider keine Bibliographie der Übersetzungen aus dem Deutschen, welche die hier relevanten vollständig erfassen würde. Zudem gibt es keine österreichische (oder deutsche) Bibliothek, die Belegstücke aller fraglichen Übersetzungen besitzt. Selbst eine Kombination der einschlägigen (und jeweils bedeutenden) Bestände von Österreichischer Nationalbibliothek, Literaturhaus Wien und Österreichischer Gesellschaft für Literatur würde immer noch ein lückenhaftes Verzeichnis ergeben. Nicht wenige Nachweise waren daher das Ergebnis glücklicher Recherchen an Orten, an denen Funde nicht unbedingt zu erwarten gewesen wären.

Eine weitere Möglichkeit, übersetzte Werke von Heimito von Doderer zu ermitteln, besteht naturgemäß darin, die Korrespondenzen zu den entsprechenden Lizenzen zu prüfen, die von den Verlagen Biederstein und C. H. Beck vergeben wurden. Obwohl diese Unterlagen von Verlagsseite vollumfänglich zur Verfügung hätten gestellt werden können, musste von einer systematischen Auswertung aus Zeitgründen abgesehen werden, da bei einer ausschnittsweisen Prüfung der Korrespondenzen deutlich wurde, dass ein nicht unerheblicher Teil der vorgesehenen Publikationen nicht realisiert worden war, weil Übersetzungslizenzen mit Ablauf der zur Verfügung stehenden Frist verfielen oder bereits zuvor zurückgegeben wurden. Unlizenzierte Übersetzungen, insbesondere von kürzeren Texten in weniger verbreitete Sprachen, die an abgelegenen Orten, als Liebhaberpublikationen und/oder in sehr geringer Auflage erschienen, hätten sich auf diesem Wege ohnehin nicht erfassen lassen.

Die „Bibliographie der in Übersetzung erschienenen Werke von Heimito von Doderer“ (vgl. Sommer 2016) verzeichnet die übersetzten Texte innerhalb der alphabetischen Abfolge der genannten Zielsprachen in der zeitlichen Reihenfolge ihres Erscheinens. (Abweichend davon werden Neuausgaben einzelner Werke jedoch unmittelbar nach der Erstausgabe angeführt - wo dies nicht sinnvoll erschien, sind die verschiedenen Auflagen durch Querverweise miteinander verbunden.) Einzelnachweise nennen nacheinander Titel der Übersetzung, Übersetzer(in), Ort, Verlag und Jahr sowie gegebenenfalls Reihe und, so vorhanden, Bandnummer. In einer neuen Zeile erscheinen der je zugehörige Identifikationscode und der deutsche Titel des übersetzten Textes. Es folgen gegebenenfalls noch Hinweise auf Erst- und Neuübersetzung sowie auf (auch geringfügige) Titel- 
änderungen. Bei auszugsweisen Übertragungen wurden auch die jeweiligen Seiten mit Textbeginn und -ende nachgewiesen.

Die übersetzten Werke sind folglich in Zielsprachen-Thesauri versammelt und bieten so zugleich je historische und quantitative Übersichten. Danach folgen eine Liste laufender Übersetzungsvorhaben und geplanter Neuauflagen sowie eine Aufstellung, in der alle übersetzten Werke durch Jahr, Zielsprache und Identifikationsnummer erschlossen werden, so dass für jeden einzelnen Text eine individuelle Übersetzungshistorie zur Verfügung gestellt wird.

Die „Bibliographie der in Übersetzung erschienenen Werke von Heimito von Doderer“ kann im Rahmen des hier zur Verfügung stehenden Raumes nicht detailliert ausgewertet werden. Es sei hier daher nur auf ein paar wesentliche Eckpunkte hingewiesen, ergänzt um einige übersetzungshistorische Auffälligkeiten und Kuriosa. Zwischenzeitlich zur Kenntnis gelangte Übersetzungen (frühere wie neu erschienene) wurden hierbei entsprechend berücksichtigt.

Die erste Übersetzung eines Werkes von Heimito von Doderer erschien 1943. Es war dies die französische Fassung seines 1940 publizierten Romans Ein Umweg. Sie trägt den Titel Sursis (vgl. Doderer 1943), wurde von Blaise Briod übertragen und kam im Pariser Verlag Plon heraus. $\mathrm{Zu}$ verdanken war das zweifellos auch den guten Kontakten nach Frankreich, die Doderers Lektor Horst Wiemer noch vor Ausbruch des Krieges, 1938 (vgl. Beck 1939), geknüpft hatte, und später, ab 1940 in Frankreich stationiert, von 1941 bis 1943 zeitweise als kommissarischer Leiter des wichtigen Verlagshauses Hachette und der Librairie Reve-Gauche (vgl. Sund 1942a, und Rebenich 2013, 413, 781), noch vertiefen konnte. Parallel zur Übersetzung von Ein Umweg wurde, wohl seit 1941, beim renommierten Pariser Verlag Gallimard eine Übertragung von Doderers Roman Ein Mord den jeder begeht (1938) vorbereitet, die allerdings - warum, ist unbekannt - weder innerhalb des vorgesehenen Zeitrahmens noch nach Ende des Zweiten Weltkriegs veröffentlicht wurde, obwohl die erste Hälfte des übersetzten Textes Gallimard am 12. Oktober 1942 vorlag (vgl. Sund 1942b).

Nach 1943 zeigt sich eine markante Lücke in der Übersetzungshistorie des Autors. Ursächlich hierfür war sicherlich, dass es nach 1945 in vielen Ländern inopportun, zumindest aber verlegerisch riskant war, zu versuchen, dem Lesepublikum deutschsprachige Literatur nahezubringen. Teilweise dürfte das Desinteresse aber auch auf den Umstand zurückzuführen sein, dass Doderer nach dem Umweg (1940) - abgesehen von der Lizenzausgabe des Romans im Wiener Luckmann-Verlag (1947) - kriegs- und kriegsfolgenbedingt nicht vor dem Jahr 1951 wieder mit Neuerscheinungen (Die Strudlhofstiege und Die erleuchteten Fenster) auf dem Buchmarkt in Erscheinung treten konnte.

Erst 1958 - sicherlich eine Auswirkung des Erfolgs des 1956 erschienenen Romans Die Dämonen, der seinem Autor unter anderem 1957 eine Titelgeschichte 
im Hamburger Nachrichtenmagazin Der Spiegel (vgl. Anon. 1957) einbrachte wurde Doderer wieder international publiziert, wobei die Anfänge sehr bescheiden waren und als solche nicht mehr als eine Tendenz anzeigen konnten. Anzuführen sind hier zwei Übertragungen ins Schwedische, eine von fünf Gedichten (vgl. Doderer 1958c) und eine der Kurzgeschichte „Ein anderer Kratki-Baschik“ (vgl. Doderer 1958a), sowie von zwei Seiten aus den Dämonen auf Italienisch (vgl. Doderer 1958b). 1959 erschien nach einer Pause von 16 Jahren neuerlich ein Text Doderers in französischer Sprache, das Vorwort zu einem Bildband über Österreich (vgl. Doderer 1959). Die erste Übersetzung ins Englische, ein Ausschnitt aus Grundlagen und Funktion des Romans wurde 1960 in Rahmen einer Veröffentlichung des P.E.N. herausgegeben (vgl. Doderer 1960).

Wirklich relevante Publikationen, also selbständig erscheinende Übersetzungen seiner Romane wurden jedoch erst in den Jahren danach publiziert, dann allerdings in kurzen Abständen und in breiter internationaler Streuung: 1961 erschienen The Demons zeitgleich bei Alfred A. Knopf in New York und bei Weidenfeld \& Nicolson in London (vgl. Doderer 1961c), die finnische Ausgabe von Ein Mord den jeder begeht (vgl. Doderer 1961b) und die italienische von Die erleuchteten Fenster (vgl. Doderer 1961a); 1962 folgte die serbokroatische (vgl. Doderer 1962), 1963 die polnische (vgl. Doderer 1963) und 1964 die englische (vgl. Doderer 1964a) Übersetzung von Ein Mord den jeder begeht; ebenfalls 1964 wurde ein Sammelband mit Übersetzungen dreier Kurzgeschichten Doderers und seines Romans Die erleuchteten Fenster ins Japanische veröffentlicht (vgl. Doderer 1964b); 1965 kamen Die Dämonen auf Französisch (vgl. Doderer 1965b) und Die Strudlhofstiege (vgl. Doderer 1965a) auf Italienisch heraus; 1966, noch zu Lebzeiten des Autors, erschienen die englische Ausgabe der Wasserfälle von Slunj (vgl. Doderer 1966a) und die spanische von Ein Mord den jeder begeht (vgl. Doderer 1966b). Da von 1961 bis 1966 auch eine ganze Reihe unselbständig erschienener Übersetzungen zu verzeichnen sind und weitere Übertragungen größerer Werke vorbereitet wurden, spricht einiges dafür, dass Heimito von Doderer in seinem letzten Lebensjahrzehnt auf dem besten Weg war, als Autor international bekannt zu werden.

Zugleich ist jedoch auch festzustellen, dass Doderer infolge kriegs- und nachkriegsbedingter Lücken in seiner Publikations- und Übersetzungshistorie mindestens 15 Jahre später als eigentlich naheliegend international reüssieren konnte. Wäre nach 1943 ein kontinuierliches Erscheinen von Übersetzungen seiner Werke möglich gewesen, hätten diese den Autor bereits ab Mitte der 1950er Jahre auch international bekannt machen können. Auch der Einstieg in den angloamerikanischen Markt mit dem dafür gänzlich ungeeigneten Opus magnum Die Dämonen, den Vincent Kling anhand von Quellen jüngst minutiös nachgezeichnet hat (vgl. Kling 2016), wäre höchstwahrscheinlich nicht misslungen, da Doderer 
bereits vor dessen Erscheinen mit Übersetzungen weniger umfangreicher und anspruchsvoller Werke ein englisches und amerikanisches Lesepublikum für sich gewinnen hätte können.

Zieht man heute, im Jahr 2018, Bilanz, so ergibt sich nur teilweise ein vorhersehbares Bild: Doderers Werke wurden insbesondere in die Weltsprachen Englisch (Das Geheimnis des Reichs, Ein Mord den jeder begeht, Die erleuchteten Fenster, Das letzte Abenteuer, Die Dämonen, Die Merowinger, Die Wasserfälle von Slunj, die Erzählungen und Kurzgeschichten sowie Teile seines essayistischen Werks [vgl. Sommer 2016, 505-511]), Französisch (Ein Mord den jeder begeht, Ein Umweg, Die erleuchteten Fenster, Das letzte Abenteuer, Die Dämonen, Die Wasserfälle von Slunj, fast alle Erzählungen und Kurzgeschichten sowie Teile seines essayistischen Werks [vgl. Sommer 2016, 512-517]) und Spanisch (Ein Mord den jeder begeht, Die Strudlhofstiege, Die Dämonen sowie die Kurzgeschichten und kleine Teile seines essayistischen Werks [vgl. Sommer 2016, 524-526]) übertragen. Gleiches gilt für die großen europäischen Nationalsprachen Italienisch (Ein Mord den jeder begeht, Die erleuchteten Fenster, Die Strudlhofstiege, Die Dämonen, eine Auswahl der Erzählungen und Kurzgeschichten sowie ein Teil des Tagebuchbandes Tangenten [vgl. Sommer 2016, 517-519]), Polnisch (Ein Mord den jeder begeht, Die Strudlhofstiege, Das letzte Abenteuer, Die Dämonen, eine Auswahl der Erzählungen und Kurzgeschichten sowie kleine Teile seines essayistischen Werks [vgl. Sommer 2016, 520-522]) und Russisch (Ein Mord den jeder begeht, Ein Umweg, Die erleuchteten Fenster, Das letzte Abenteuer, Die Wasserfälle von Slunj, eine Auswahl der Erzählungen und Kurzgeschichten sowie kleine Teile seines essayistischen Werks [vgl. Sommer 2016, 522f.]).

Zugleich gibt es aber auch überraschende Schwerpunkte bei Sprachen mit geringer Verbreitung. Insbesondere zu erwähnen ist hier das Estnische. Bereits 1970 erschien ein Sammelband mit den Erzählungen „Ein anderer KratkiBaschik“, „Unter schwarzen Sternen“ und dem Divertimento No VII: Die Posaunen von Jericho (vgl. Doderer 1970); es folgten Ein Mord den jeder begeht (vgl. Doderer 1974) und Die Strudlhofstiege (vgl. Doderer 2008b) sowie Die Merowinger (vgl. Doderer 2012), was sicherlich dem besonderen Engagement des Übersetzers Mati Sirkel zu danken ist.

Am weitesten in Übersetzung verbreitet ist aktuell Doderers Roman Ein Mord den jeder begeht, der bis einschließlich 2017 in dreizehn Sprachen übersetzt wurde (vgl. Sommer 2016, 533). Danach folgt eine Erzählung, „Die Peinigung der Lederbeutelchen“, von der Ausgaben in elf Fremdsprachen (Chinesisch, Englisch, Französisch, Georgisch, Japanisch, Polnisch, Portugiesisch, Russisch, Tschechisch, Ungarisch und Weißrussisch [vgl. Sommer 2016, 531]) vorliegen. Erst an dritter Stelle steht mit Übertragungen in zehn Sprachen Doderers bekanntester Roman Die Strudlhofstiege (vgl. Sommer 2016, 531f.). 
Wenn man sich die Übersetzungsgeschichte der genannten Werke ansieht, ergibt sich freilich ein teils doch überraschendes bis seltsames Bild. Wie ist es etwa zu erklären, dass der Mord zuerst gerade ins Finnische (vgl. Doderer 1961b), danach ins Kroatische (vgl. Doderer 1962) und Polnische (vgl. Doderer 1963) und erst dann ins Englische (vgl. Doderer 1964a) und Spanische (vgl. Doderer 1966b) übertragen wurde? Wie kam eine solche Abfolge zustande? Ich vermute, weniger aufgrund eines geplanten Vorgehens des Biederstein-Verlags als infolge der Vermittlungsarbeit von Kulturinstituten - das persönliche Interesse einzelner Übersetzer an Doderer sollte man hier wohl ebenfalls nicht unterschätzen. Auch die weitere Verteilung ist ungewöhnlich: Es schlossen sich an: Übertragungen in Afrikaans (vgl. Doderer 1968) und ins Estnische (vgl. Doderer 1974), danach erst gab es Übersetzungen ins Italienische (vgl. Doderer 1983) und Französische (vgl. Doderer 1986). Hinzu kamen noch Übertragungen ins Bulgarische (vgl. Doderer 1985) und Rumänische (vgl. Doderer 1990a); relativ spät erschien der Mord auf Niederländisch (vgl. Doderer 2001) und Russisch (vgl. Doderer 2006). Besonders zu erwähnen sind hier noch eine Neuübersetzung ins Spanische (vgl. Doderer 1995) und der Umstand, dass der Roman sehr viel früher auf Afrikaans vorlag als im nächstverwandten Niederländisch.

Wenig überrraschend ist allerdings, dass Übersetzungen von Ein Mord den jeder begeht eher und häufiger erschienen sind als jene der Strudlhofstiege. Doderers bekanntester Roman ist nicht nur sehr viel schwerer zu übersetzen, er hat mit 900 Seiten zudem etwa den zweieinhalbfachen Umfang des Mord. Das unternehmerische Risiko für einen Verlag ist damit auch deutlich größer. Betrachtet man die Übersetzungeschichte der Strudlhofstiege genauer, so findet sich zuerst eine Übertragung ins Italienische (vgl. Doderer 1965a). Erst 14 Jahre und später erschienen weitere Übersetzungen: ins Polnische (vgl. Doderer 1979), Spanische (vgl. Doderer 1981) und Bulgarische (vgl. Doderer 1984). Wiederum mit zeitlichem Abstand folgten Ausgaben der Strudlhofstiege auf Slowakisch (vgl. Doderer 1990b), Slowenisch und Ungarisch (vgl. Doderer 1994b, und Doderer 1994a), auf Estnisch und Niederländisch (vgl. Doderer 2008b, und Doderer 2008a) sowie auf Kroatisch (vgl. Doderer 2013/2014). Doderers bekanntester Roman liegt bisher mithin nur in einer einzigen Weltsprache, nämlich Spanisch, vor und zudem in einer Reihe von Sprachen mit mittlerer und geringer Verbreitung - bedauerlicherweise existieren jedoch weder Übersetzungen ins Englische noch ins Französische oder Russische. Diese Konstellation dürfte sich in den nächsten Jahren jedoch deutlich verändern, da derzeit parallel gleich an vier Übersetzungen der Strudlhofstiege gearbeitet wird: ins Englische (Vincent Kling), ins Französische (Pierre Deshusses), ins Tschechische (Ondřej Sekal) und ins Türkische (Etem Levent Bakaç).

Damit endet dieser kurze Überblick - zum Schluss noch ein Kuriosum der besonderen Art, das mir (und sicher nicht nur mir) Rätsel aufgibt: Gelegentlich wur- 
den (und werden) Texte auch mehrmals in eine Zielsprache übersetzt, das ist nichts Ungewöhnliches. Wie aber lässt es sich erklären, dass Doderers Erzählung „Zwei Lügen oder Eine antikische Tragödie auf dem Dorfe“ binnen 24 Jahren (vgl. Doderer 1978, Doderer 1987 bzw. Doderer 1996, und Doderer 2002) nacheinander dreimal von verschiedenen Übersetzern (Wladimir Zaridse, Giorgi Matschutadse und Tengis Khatschapuridse) ausgerechnet ins Georgische übertragen und zudem insgesamt viermal publiziert wurde? Das gibt mir doch zu denken und uns allen die Frage mit auf den Weg: Was mag georgische Übersetzer ausgerechnet an dieser Erzählung so fasziniert haben?

\section{Literaturverzeichnis}

Anon. „Doderer. Der Spätzünder“. Der Spiegel 11.23 (1957): 53-58.

Beck, Heinrich an Heimito von Doderer. Brief vom 20. Januar 1939. Literaturarchiv der Österreichischen Nationalbibliothek, Wien (LIT), Autogr. 523/51-6.

Doderer, Heimito von. Sursis. Roman. Trad. de l'allemand par Blaise Briod. Paris: Plon, 1943.

Doderer, Heimito von. „Den nye Kratki-Baschik“. Tysk Samtid. Hg. Guenter Klingmann. Stockholm: LTs Förl., 1958a. 35-47.

Doderer, Heimito von. „Due pagine dei ,Demoni“. La gerente del caffè Kaunitz / Una serata dal principe Croix“. Traduzione autorizzata di Margaret e Gianfranco Contini. L'Approdo letterario 4.3 (1958b): 81f.

Doderer, Heimito von. „Ur VÄG I MÖRKNINGEN“. Sverige - Tyskland 21.1 (1958c): $13 f$.

Doderer, Heimito von. „[Introduction]“. Autriche. Texte de Heimito von Doderer. Photos de Toni Schneiders. Paris: Braun, 1959 (Collection Atlantis). 5-23.

Doderer, Heimito von. „Principles and Function of the Novel“. XXX. Kongress des Internationalen P.E.N Frankfurt am Main 19. bis 25. Juli 1959. Schöne Literatur im Zeitalter der Wissenschaft I Imaginative Literature in the Age of Science / La création littéraire a l'age de la science. Bericht / Report / Compte-rendu. Frankfurt/M. und Berlin: Propyläen, 1960 (Kongreß des Internationalen PEN; 30). 56-68.

Doderer, Heimito von. Le finestre illuminate ovvero Come il consigliere Julius Zihal divenne uomo. Torino: Einaudi, 1961a (I coralli; 137).

Doderer, Heimito von. Murha jon ka jokainen tekee. Suomentanut Eila Nisonen. Porvoo: WSOY, 1961b.

Doderer, Heimito von. The Demons. Transl. from the German by Richard and Clara Winston. New York, NY: Alfred A. Knopf, 1961c.

Doderer, Heimito von. Ubojstvo koje svatko počinja. Preveo: Zvonimir Golob. Zagreb: Lykos, 1962 (Vikend; 5).

Doderer, Heimito von. Każdy może być morderca. Przełożyła Kazimiera Itłakowiczówna. Posłowie Roman Karst. Warszawa: Państwowy Instytut Wydawniczy, 1963.

Doderer, Heimito von. Every Man a Murderer. Transl. from the German by Richard and Clara Winston. New York, NY: Alfred A. Knopf, 1964a.

Doderer, Heimito von. Mado no hi. Tōkyō: Hakusuisha, 1964b (Atarashii sekai no bungaku; 11).

Doderer, Heimito von. La scalinata. Traduzione di Ervino Pocar. Presentazione di Margaret Contini. Torino: Einaudi, 1965a. 
Doderer, Heimito von. Les démons. D'après la chronique du Chef de division Geyrenhoff. Traduit de l'allemand par Robert Rovini. Paris: Gallimard, 1965b (Du Monde Entier; 387). Doderer, Heimito von. The Waterfalls of Slunj. Transl. by Eithne Wilkins and Ernst Kaiser. New York, NY: Harcourt, Brace \& World, 1966a.

Doderer, Heimito von. Un asesinato que comete cualquiera. Barcelona: Plaza \& Janés, 1966b.

Doderer, Heimito von. 'n Moord wat elkeen pleeg. Johannesburg: Boek Mosaiek, 1968 (Boek Mosaiek; 33).

Doderer, Heimito von. Jeeriku pasunad. Saksa keelest tõlkinud Ain Kaalep. Tallinn: Kirjastus Perioodika, 1970 (Loomingu raamatukogu; 43).

Doderer, Heimito von. Igaühest võib saada mõrvar. Romaan. Saksa keelest tõlkinud Armand Tungal. Tallinn: Eesti Raamat, 1974.

Doderer, Heimito von. „Ori tquili, anu ert'i antikuri tragedia sop'elshi“. Germanulidan k'art'ulad t’argmna Wladimir Zaridse. Pirveli skhivi 13 (1978): 111-119.

Doderer, Heimito von. Schody Strudlhofu albo Melzer i gtębia lat. Przełożył Stawomir Błaut. Posłowie napisat Henryk Bereza. Poznań: Wydawnictwo Poznańskie, 1979.

Doderer, Heimito von. Las Escaleras de Strudlhof. Traducción e introducción de José Miguel Sáenz. Barcelona: Destino, 1981 (Áncora y Delfín; 541).

Doderer, Heimito von. L'occasione di uccidere. Traduzione di Aldo Busi. Con una nota di Claudio Magris. Milano: Garzanti, 1983.

Doderer, Heimito von. Melcer i mădrostta na godinite. Roman. Prevede ot nemski Velizar Bonev. Plovdiv: Izdatelstvo Christo G. Danov, 1984.

Doderer, Heimito von. Ubijstvo, koeto vseki izvursava. Prevela ot nemski Krasimira Angelova. Sofija: Narodna Kultura, 1985.

Doderer, Heimito von. Un meurtre que tout le monde commet. Trad. de l'allemand par Pierre Deshusses. Paris u. Marseille: Rivages, 1986.

Doderer, Heimito von. „Ori tquili, anu antikuri tragedia sop’lad“. Germanulidan k’art’ulad t’argmna Giorgi Matschutadse. Ritsa 13.2 (1987): 244-254.

Doderer, Heimito von. Drumul lui Conrad Castiletz. Trad. de Octavian Nicolae. Bucureşti: Ed. Univers, 1990a.

Doderer, Heimito von. Strudlhofské schody alebo Melcer a hlbina rokov. Preložila a doslov napísala Viera Juríčkova. Bratislava: Tatran, 1990b (Svetová tvorba; 202).

Doderer, Heimito von. A Strudlhof-lépcső. Fordította Tandori Dezső. A fordítást az eredetirel egyberetette Csordás Gábor. Pécs: Jelenkor Kiadó, 1994a.

Doderer, Heimito von. Strudlhofovo stopnišče ali Melzer in globina let. Prevedla Stanka Rendla. Ljubljana: Mihelač, 1994b (Zbirka Svetovni klasiki; 33).

Doderer, Heimito von. Un asesinato que todos cometemos. Traducido del alemán por Adan Kovacsics. Barcelona: Muchnik, D. L., 1995.

Doderer, Heimito von. „Ori tquili, anu antikuri tragedia sop’lad“. Germanulidan k’art'ulad t’argmna Giorgi Matschutadse. Kavkasioni (19. 12. 1996): 6f.

Doderer, Heimito von. leder mens een moordenaar. Vertaald door Nelleke van Maaren. Amsterdam und Antwerpen: Uitgeverij Atlas, 2001.

Doderer, Heimito von. „Ori tquili anu antikuri tragedia sop’lad“. Avstriuli novelebi / Österreichische Novellen. Germanulidan t'argmna t'engiz khachapuridzem / Aus dem Deutschen ins Georgische übersetzt von Tengis Khatschapuridse. Tbilissi: FAVORIT, 2002. 34-46.

Doderer, Heimito von. Ubijstvo, kotoroe soveršaet každyj. Roman. Per. s nemeckogo S. E. Šlapoberskoj. St. Petersburg: Izdatel'stvo Ivana Limbacha, 2006. 
Doderer, Heimito von. De Strudlhoftrappen of Melzer en de diepte der jaren. Vertaald door Nelleke van Maaren. Met een nawoord van Hans Driessen. Amsterdam u. Antwerpen: Uitgeverij Atlas, 2008a.

Doderer, Heimito von. Strudlhofi trepp ehk Melzer ja aastate sügavus. Romaan. Saksa keelest tõlkinud Mati Sirkel. Tallinn: Eesti Raamat, 2008b.

Doderer, Heimito von. Merovingid ehk totaalne sugu. Romaan. Saksa keelest tõlkinud Mati Sirkel. Tallinn: Eesti Keele Sihtasutus, 2012.

Doderer, Heimito von. Strudlhofstiege ili Melzer i dubina godina koje prolaze. Roman. Prijevod s njemačkog Andy Jelčić. Zagreb: Leykam international, 2013 [Bd. 1] und 2014 [Bd. 2].

Kling, Vincent. „,Our Colossal Failure‘: Heimito von Doderer’s Debut in English“. DodererGespräche. Mit einer Grundlegung zu Paul Elbogen. Hg. Achim Hölter, Gerald Sommer, Sarah Kohl, Robert Walter-Jochum, Kirk Wetters und François Grosso. Würzburg: Königshausen \& Neumann, 2016 (Schriften der Heimito von Doderer-Gesellschaft; 7). 83-92.

Rebenich, Stefan. C. H. Beck 1763 - 2013. Der kulturwissenschaftliche Verlag und seine Geschichte. München: C. H. Beck, 2013.

Sommer, Gerald. „Bibliographie der in Übersetzung erschienenen Werke von Heimito von Doderer“. Doderer-Gespräche. Mit einer Grundlegung zu Paul Elbogen. Hg. Achim Hölter, Gerald Sommer, Sarah Kohl, Robert Walter-Jochum, Kirk Wetters und François Grosso. Würzburg: Königshausen \& Neumann, 2016 (Schriften der Heimito von Doderer-Gesellschaft; 7). 503-536.

Sund, Georg an Heimito von Doderer. Brief vom 31. März 1942a. LIT, Autogr. 523/51-9.

Sund, Georg an Heimito von Doderer. Brief vom 23. Oktober 1942b. LIT, Autogr. 523/51-10.

Gerald Sommer, geb. 1963 in Karlsruhe. Freier Autor, Redakteur und Herausgeber in Berlin. 1999 Promotion über Heimito von Doderers ,technische Mittel“. Mitbegründer und (seit 2008) Vorsitzender der Heimito von Doderer-Gesellschaft sowie Herausgeber ihrer Schriften-Reihe. Zahlreiche Publikationen zu Doderer sowie Editionen aus dem Nachlass des Autors. 
